\title{
Cleavage, Down-regulation and Aggregation of Serum Amyloid A
}

\author{
Wenhua Wang, ${ }^{*}, \uparrow, \ddagger$ Prabir Khatua, ${ }^{*, \dagger, \ddagger}$ and Ulrich H.E. Hansmann ${ }^{*, \dagger}$ \\ $\dagger$ Dept. of Chemistry 83 Biochemistry, University of Oklahoma, Norman, OK 73019, USA \\ $\ddagger W$.W. and P.K. contributed equally to this work \\ E-mail: wenhuawang@ou.edu; prabir@ou.edu; uhansmann@ou.edu
}


Table SF1: Solvent Accessible Surface Area $(\langle S A S A\rangle)$ per residue averaged over all chains in the $\mathrm{SAA}_{1-76}$ and $\mathrm{SAA}_{1-104}$ hexamers. We show both the values for the individual trajectories and for the resulting averages (with standard deviation listed in parenthesis). For comparison we list also the respective values for the free monomers.

\begin{tabular}{cccc}
\hline \hline \multicolumn{2}{c}{ system } & Hexamer & Monomer \\
\hline & Run-1 & 55.7 & 77.6 \\
& Run-2 & 55.2 & 72.7 \\
SAA $_{1-76}$ & Run-3 & 55.7 & 79.1 \\
& Average & $55.5(0.3)$ & $76(3)$ \\
\hline & Run-1 & 51.2 & 69.0 \\
& Run-2 & 50.8 & 68.7 \\
SAA $_{1-104}$ & Run-3 & 51.6 & 69.1 \\
& Average & $51.2(0.4)$ & $69.2(0.4)$ \\
\hline
\end{tabular}


(a)

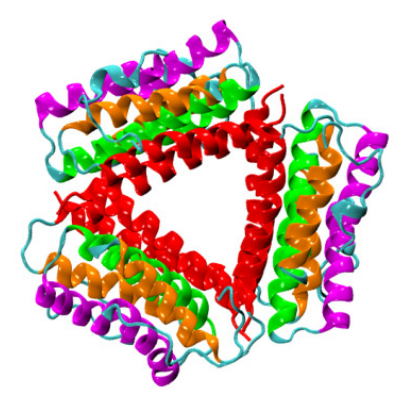

(d)

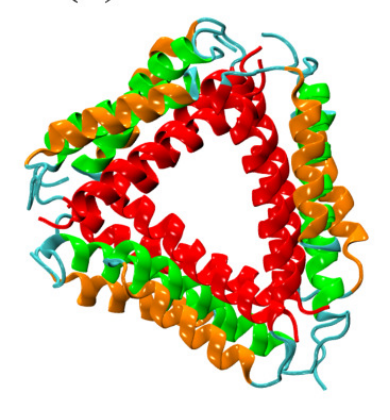

(b)

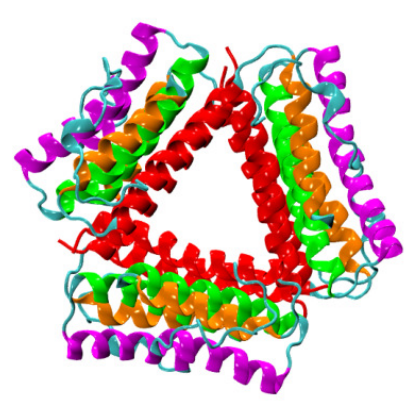

(e)

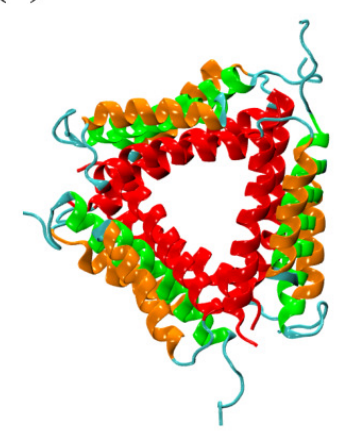

(c)

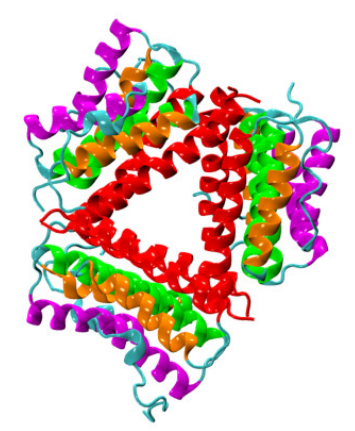

(f)

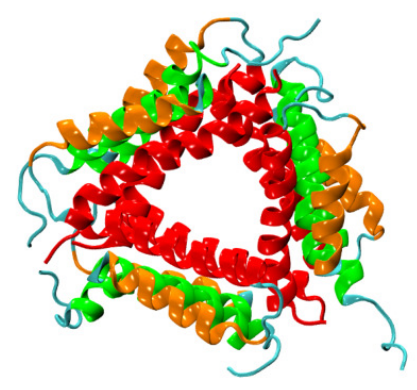

Figure SF1: The snapshots of (a-c) $\mathrm{SAA}_{1-104}$ and (d-f) $\mathrm{SAA}_{1-76}$ hexamers as obtained at the end of each of the three trajectories. The trajectories are shown as follows: first column (Run-1), second column (Run-2), and third column (Run-3). Color coding of the helices: helix-I (red), helix-II (orange), helix-III (green), and helix-IV (magenta). 


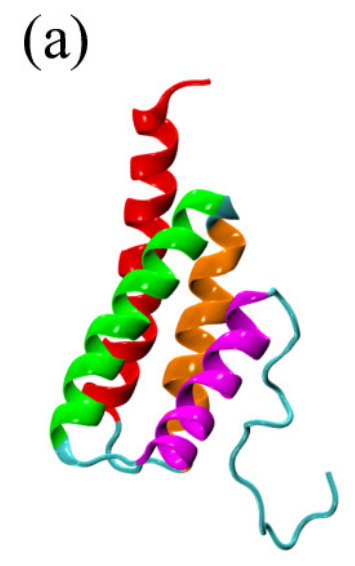

(b)

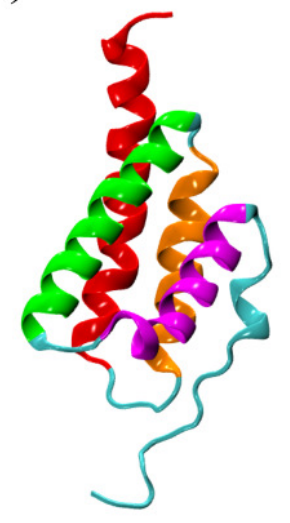

(d)

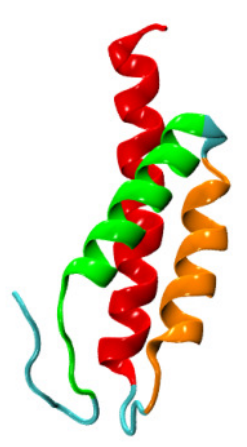

(e)

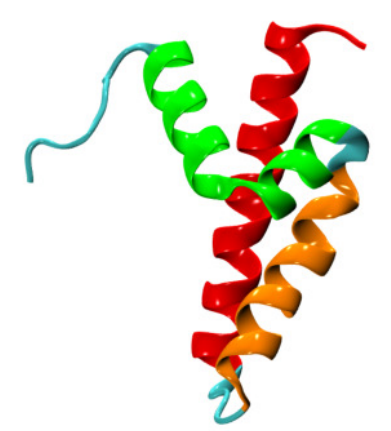

(c)

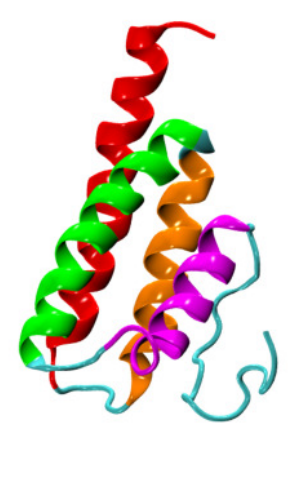

(f)

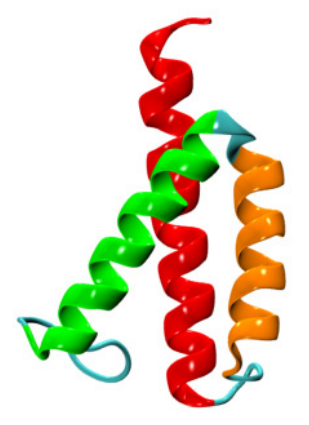

Figure SF2: The snapshots of (a-c) $\mathrm{SAA}_{1-104}$ and (d-f) $\mathrm{SAA}_{1-76}$ monomers as obtained at the end of each of the three trajectories. The trajectories and color coding of the helices are same as that of Figure 1. 

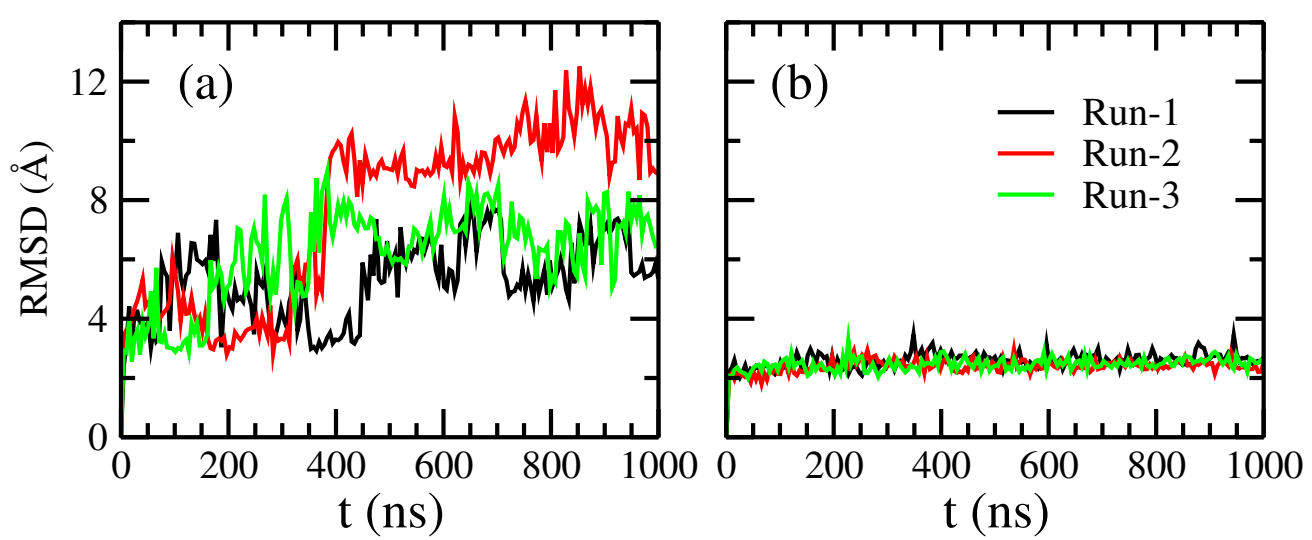

Figure SF3: Time evolution of the root mean square deviation (RMSD) of isolated (a) $\mathrm{SAA}_{1-76}$ and (b) $\mathrm{SAA}_{1-104}$ monomers, taking into account all non-hydrogen atoms in the first 76 residues. Data are shown for all three trajectories of each system. The RMSD values are calculated with respect to the corresponding start configurations. 

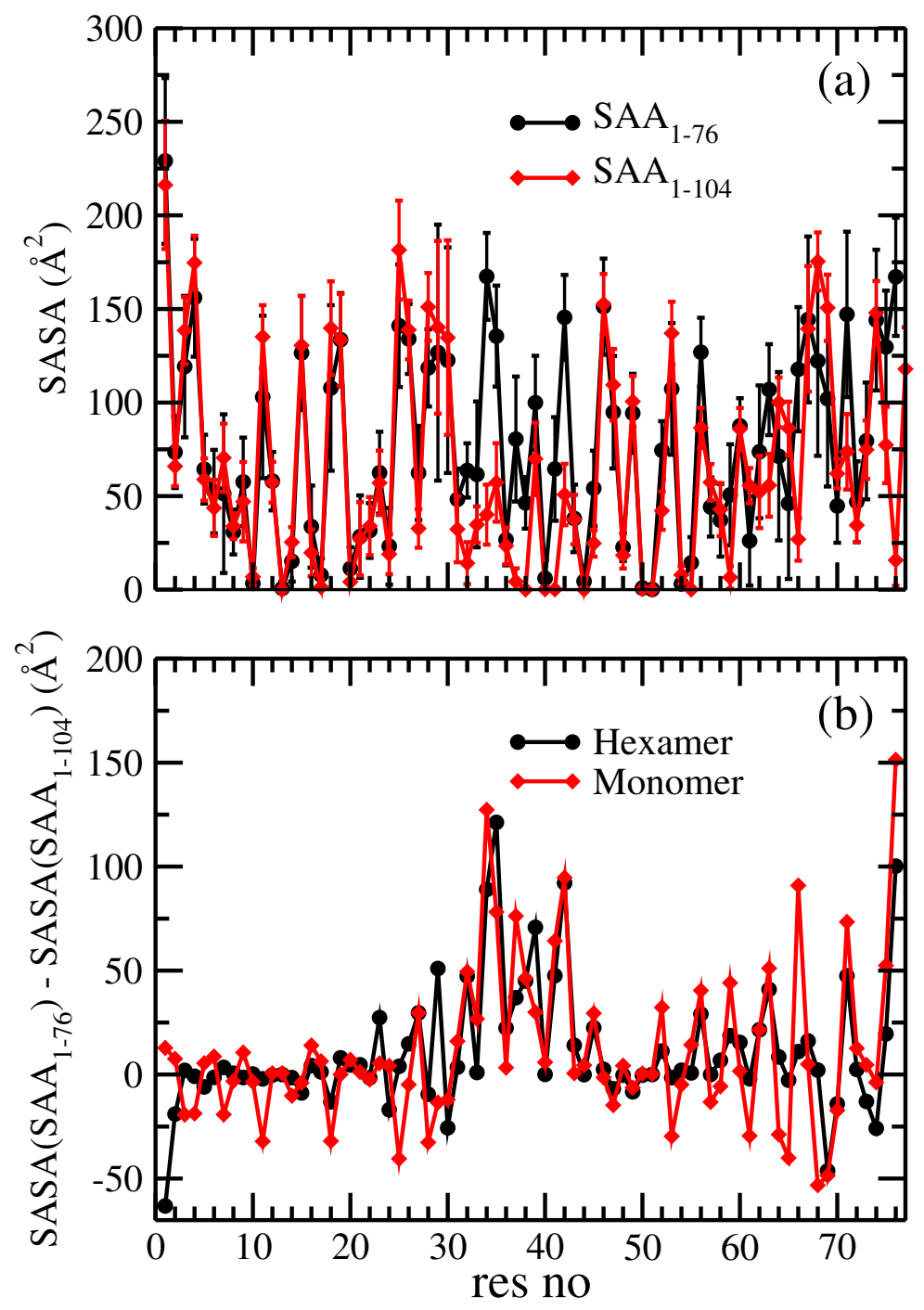

Figure SF4: (a) Average residue-wise solvent accessible surface area (SASA) of $\mathrm{SAA}_{1-76}$ and $\mathrm{SAA}_{1-104}$ monomers as calculated over the last $500 \mathrm{~ns}$ of each of the three trajectories of each system. Data are shown only for the first 76 residues. In (b) we show for each residue the difference $\triangle S A S A=S A S A\left(S A A_{1-76}\right)-S A S A\left(S A A_{1-104}\right)$ calculated either for the isolated monomer or measured in the corresponding hexamers. 